\title{
Equidistribution for nonuniformly expanding dynamical systems, and application to the almost sure invariance principle
}

\author{
Alexey Korepanov \\ Mathematics Institute, University of Warwick, Coventry, CV4 7AL, UK
}

January 13, 2017 (updated October 21, 2017)

\begin{abstract}
Let $T: M \rightarrow M$ be a nonuniformly expanding dynamical system, such as logistic or intermittent map. Let $v: M \rightarrow \mathbb{R}^{d}$ be an observable and $v_{n}=\sum_{k=0}^{n-1} v \circ T^{k}$ denote the Birkhoff sums. Given a probability measure $\mu$ on $M$, we consider $v_{n}$ as a discrete time random process on the probability space $(M, \mu)$.

In smooth ergodic theory there are various natural choices of $\mu$, such as the Lebesgue measure, or the absolutely continuous $T$-invariant measure. They give rise to different random processes.

We investigate relation between such processes. We show that in a large class of measures, it is possible to couple (redefine on a new probability space) every two processes so that they are almost surely close to each other, with explicit estimates of "closeness".

The purpose of this work is to close a gap in the proof of the almost sure invariance principle for nonuniformly hyperbolic transformations by Melbourne and Nicol.
\end{abstract}

\section{Introduction}

Suppose that $T: M \rightarrow M$ is a dynamical system, and $v: M \rightarrow \mathbb{R}^{d}$ is an observable. Let $v_{n}=\sum_{k=0}^{n-1} v \circ T^{k}$ denote the Birkhoff sums. Given a probability measure $\mu$ on $M$, let $\left(v_{n}, \mu\right)$ denote the discrete time random process given by $v_{n}$ on the probability space $(M, \mu)$.

In the study of statistical properties of $v_{n}$, such as the the central limit theorem, various choices for $\mu$ come up naturally, giving rise to different random processes.

For example, if $M=[0,1]$ and $T$ is a nonuniformly expanding map as in Young [23] such as intermittent or logistic with a Collet-Eckmann parameter, then $\mu$ may be (a) the Lebesgue measure, (b) the absolutely continuous invariant probability measure (a.c.i.p.), (c) the a.c.i.p. for the associated induced map (see Section 21).

The interest in the Lebesgue measure comes from physics: it is a natural choice of initial condition. The a.c.i.p. has an important advantage over the Lebesgue measure: if 
$\mu$ is the a.c.i.p., then the increments of the process $\left(v_{n}, \mu\right)$ are stationary. It is standard to prove and state limit theorems in terms of the a.c.i.p.

The measure in (c) appears in a widely used technical argument, when $T$ is reduced by a time change (inducing) to a uniformly expanding map, which may be easier to work with. Then statistical properties of the induced map are used to prove results on the original map.

We explore the relation between processes defined with respect to different measures. Our motivation is the study of almost sure approximations by Brownian motion.

Definition 1.1. We say that $v_{n}$ satisfies the Almost Sure Invariance Principle (ASIP), if without changing the distribution, $\left\{v_{n}, n \geq 0\right\}$ can be redefined on a new probability space with a Brownian motion $W_{t}$, such that with some $\beta<1 / 2$,

$$
v_{n}=W_{n}+o\left(n^{\beta}\right) \quad \text { almost surely. }
$$

The ASIP is a strong statistical property, it implies the central limit theorem (CLT) and the law of iterated logarithm (LIL), which in one dimension take form

$$
\mathbb{P}\left(\frac{v_{n}}{\sqrt{n}} \in[a, b]\right) \stackrel{n \rightarrow \infty}{\longrightarrow} \frac{1}{\sqrt{2 \pi \sigma^{2}}} \int_{a}^{b} e^{-\frac{x^{2}}{2 \sigma^{2}}} d x \quad \text { for all } a \leq b
$$

and

$$
\limsup _{n \rightarrow \infty} \frac{v_{n}}{\sqrt{n \log \log n}}=\sqrt{2} \sigma \quad \text { almost surely. }
$$

The ASIP also implies functional versions of the CLT and the LIL as well as other laws, see Philipp and Stout [18, Chapter 1].

Melbourne and Nicol [16, 17] proved

Theorem 1.2. Suppose that $T$ is nonuniformly expanding with return times in $L^{p}, p>2$ (see Section 2 for definitions) with an absolutely continuous invariant probability measure $\rho$. If $v: M \rightarrow \mathbb{R}^{d}$ is a Hölder continuous continuous observable with $\int_{M} v d \rho=0$, then the process $v_{n}=\sum_{k=0}^{n-1} v \circ T^{k}$, defined on a probability space $(M, \rho)$, satisfies the ASIP.

Remark 1.3. Following the approach of [2, 21], the ASIP for nonuniformly expanding systems extends to a large class of nonuniformly hyperbolic systems which satisfy the hypotheses of Young [22, for example Sinai billiards or Hénon maps. See [16, Lemma 3.2].

Later Gouëzel discovered a gap in [16, 17]: what Melbourne and Nicol actually proved is the ASIP for a different starting measure, the one invariant invariant under the induced map. A similar issue appears in Denker and Philipp [6], though they do not claim the ASIP for the invariant measure. Even though there is a close relation between the two measures, the argument relating the ASIP-s was missing. The main goal of this paper is to fill this gap.

Remark 1.4. Despite the gap, the usual corollaries of the ASIP (such as the functional central limit theorem and functional law of iterated logarithm) can be obtained from [16, 17], as it is done in [6].

Remark 1.5. Besides [16, 17], there are other results which cover nonuniformly hyperbolic systems, but only partially: 
- Chernov [3]: scalar ASIP for dispersing billiards.

- Gouëzel [8]: vector valued ASIP for dynamical systems with an exponential multiple decorrelation assumption (includes dispersing billiards).

- Cuny and Merlevède [5]: scalar ASIP for reverse martingale differences (applies to nonuniformly expanding maps, see [14]).

Problems which are only covered by [16] and [17] include the vector valued ASIP for maps with slower than exponential rate of decay of correlations, such as the intermittent family [15].

We work in the setting where $T$ is a nonuniformly expanding map (as in [23]) and $v_{n}=\sum_{k=0}^{n-1} v \circ T^{k}$ are Birkhoff sums. Given two probability measures $\mu$ and $\rho$, we compare the random processes $X_{n}=\left(v_{n}, \mu\right)$ and $Y_{n}=\left(v_{n}, \rho\right)$.

Our main result is that if $v$ is bounded, then in a large class of probability measures, it is possible to redefine $\left\{X_{n}, n \geq 0\right\}$ and $\left\{Y_{n}, n \geq 0\right\}$ on a new probability space so that $Z=\sup _{n \geq 0}\left|X_{n}-Y_{n}\right|$ is finite almost surely.

Remark 1.6. Technically, the statement above means that there exists a probability space $(\Omega, \mathbb{P})$, supporting processes $X_{n}^{\prime}, Y_{n}^{\prime}$, such that:

- $\left\{X_{n}, n \geq 0\right\}$ is equal in distribution to $\left\{X_{n}^{\prime}, n \geq 0\right\}$,

- $\left\{Y_{n}, n \geq 0\right\}$ is equal in distribution to $\left\{Y_{n}^{\prime}, n \geq 0\right\}$,

- $Z^{\prime}=\sup _{n \geq 0}\left|X_{n}^{\prime}-Y_{n}^{\prime}\right|$ is finite almost surely.

In addition, we estimate the tails of $Z$ (i.e. $\mathbb{P}(Z \geq a)$ for $a \geq 0$ ) in terms of $|v|_{\infty}$ and parameters of $T$ such as distortion bound and asymptotics of return times.

For a fixed $n \geq 0$, we estimate the distance between $X_{n}$ and $Y_{n}$ in Lévy-Prokhorov and Wasserstein metrics. We expect such estimates to be useful for families of dynamical systems as in [12].

Remark 1.7. Our approach is in many ways similar to the Coupling Lemma for dispersing billiards [4, Lemma 7.24], due to Chernov, Dolgopyat and Young. Also, after the first version of this paper was circulated, the author was made aware that some of the techniques are analogous to those in Zweimüller [24]. Notably, our disintegration (5) corresponds to Zweimüller's regenerative partition of unity.

The paper is organized as follows. In Section 2 we give the definition of nonuniformly expanding maps and state our results. In Section 3 we present some applications, including the ASIP in Subsection 3.4. Section 4 contains the proofs.

\section{Abstract setup and results}

\subsection{Nonuniformly expanding maps}

We use notation $\mathbb{N}=\{1,2, \ldots\}$ and $\mathbb{N}_{0}=\mathbb{N} \cup\{0\}$.

Let $(M, d)$ be a metric space with a Borel probability measure $m$ and $T: M \rightarrow M$ be a nonsingular transformation. We assume that there exists $Y \subset M$ with $m(Y)>0$ and $\operatorname{diam} Y<\infty$, an at most countable partition $\alpha$ of $Y$ (modulo a zero measure set) and $\tau: Y \rightarrow \mathbb{N}$ with $\int_{Y} \tau d m<\infty$ such that for every $a \in \alpha$,

- $m(a)>0$,

- $\tau$ assumes a constant value $\tau(a)$ on $a$, 
- $T^{\tau(a)} a \subset Y$.

Let $F: Y \rightarrow Y, F y=T^{\tau(y)} y$. We require that there are constants $\lambda>1, \hat{K} \geq 0$ and $\eta \in(0,1]$, such that for each $a \in \alpha$ and $x, y \in a$ :

- $F$ restricts to a (measure-theoretic) bijection from $a$ onto $Y$,

- $d(F x, F y) \geq \lambda d(x, y)$,

- the inverse Jacobian $\zeta_{m}=\frac{d m}{d m \circ F}$ of $F$ has bounded distortion:

$$
\left|\log \zeta_{m}(x)-\log \zeta_{m}(y)\right| \leq \hat{K} d(F x, F y)^{\eta} .
$$

We call such maps $T$ nonuniformly expanding. We refer to $F$ as induced map and to $\tau$ as return time function. The class of nonuniformly expanding maps includes logistic maps at Collet-Eckmann parameters, intermittent maps [23] and Viana maps.

To simplify the exposition, we assume that $\operatorname{diam} Y \leq 1$ and $\eta=1$. The general case can be always reduced to this by replacing the metric $d$ with $d^{\prime}$ given by $d^{\prime}(x, y)=$ $c d(x, y)^{\eta}$, where $c$ is a sufficiently small constant.

It is standard that there exists a unique $F$-invariant absolutely continuous probability measure $\mu$ on $Y$. Let $\zeta=\frac{d \mu}{d \mu \circ F}$. By [13, Propositions 2.3 and 2.5],

$$
K^{-1} \leq \frac{d \mu}{d m} \leq K \quad \text { and } \quad|\log \zeta(x)-\log \zeta(y)| \leq K d(F x, F y)
$$

for all $x, y \in a, a \in \alpha$, where $K$ is a constant which depends continuously (only) on $\lambda$ and $\hat{K}$. Where convenient, we view $\mu$ as a measure on $M$ supported on $Y$.

For a function $\phi: Y \rightarrow \mathbb{R}$ denote

$$
|\phi|_{\infty}=\sup _{x \in Y}|\phi(x)|, \quad|\phi|_{d}=\sup _{x \neq y \in Y} \frac{|\phi(x)-\phi(y)|}{d(x, y)} \quad \text { and } \quad\|\phi\|_{d}=|\phi|_{\infty}+|\phi|_{d} .
$$

For $\phi: Y \rightarrow(0, \infty)$, denote $|\phi|_{d, \ell}=|\log \phi|_{d}$.

\subsection{Coupling of processes}

Fix a constant $R^{\prime}>K \lambda /(\lambda-1)$.

Definition 2.1. We call a probability measure $\rho$ on $M$ regular if it is supported on $Y$ and $d \rho=\phi d \mu$, where $\phi: Y \rightarrow[0, \infty)$ satisfies $|\phi|_{d, \ell} \leq R^{\prime}$.

Definition 2.2. We say that a probability measure $\rho$ on $M$ is forward regular, if it allows a disintegration

$$
\rho=\int_{E} \rho_{z} d \varkappa(z)
$$

where $(E, \varkappa)$ is a probability space and $\left\{\rho_{z}\right\}$ is a measurable family of probability measures on $M$, and there exists a function $r: E \rightarrow \mathbb{N}_{0}$ such that $T_{*}^{r(z)} \rho_{z}$ is a regular measure for each $z$. We refer to $r$ as a jump function.

Define $s: M \times M \rightarrow \mathbb{N}_{0} \cup\{\infty\}$,

$$
s(x, y)=\inf \left\{\max \{k, n\}: k, n \geq 0, T^{k} x=T^{n} y\right\} .
$$

Note that if $s(x, y)<\infty$, then the trajectories $T^{k} x$ and $T^{k} y, k \geq 0$ coincide up to a time shift and possibly different beginnings. 
Theorem 2.3. Suppose that a probability measure $\rho$ on $M$ is forward regular. Then there exists a probability measure $\hat{\rho}$ on $M \times M$ with marginals $\rho$ and $\mu$ on the first and second components respectively such that $s$ is finite $\hat{\rho}$-almost surely.

In addition, with $(E, \varkappa)$ and $r$ as in Definition 2.2,

(a) (Weak polynomial moments) If $\varkappa(r \geq n) \leq C_{\beta} n^{-\beta}$ and $\mu(\tau \geq n) \leq C_{\beta} n^{-\beta}$ for all $n \geq 1$ with some constants $\beta>1$ and $C_{\beta}>0$, then

$$
\hat{\rho}(s \geq n) \leq C n^{-\beta} \quad \text { for all } n>0,
$$

where the constant $C$ depends continuously (only) on $\lambda, K, R^{\prime}, \beta$ and $C_{\beta}$.

(b) (Strong polynomial moments) If $\int r^{\beta} d \varkappa \leq C_{\beta}$ and $\int \tau^{\beta} d \mu \leq C_{\beta}$ with some constants $\beta>1$ and $C_{\beta}>0$, then

$$
\int s^{\beta} d \hat{\rho} \leq C
$$

where the constant $C$ depends continuously (only) on $\lambda, K, R^{\prime}, \beta$ and $C_{\beta}$.

(c) (Exponential and stretched exponential moments) If $\varkappa(r \geq n) \leq C_{\alpha, \gamma} e^{-\alpha n^{\gamma}}$ and $\mu(\tau \geq n) \leq C_{\alpha, \gamma} e^{-\alpha n^{\gamma}}$ for all $n$ with some constants $\alpha>0, \gamma \in(0,1], C_{\alpha, \gamma}>0$, then

$$
\hat{\rho}(s \geq n) \leq C e^{-A n^{\gamma}} \quad \text { for all } n>0,
$$

where the constants $C>0$ and $A>0$ depend continuously (only) on $\lambda, K, R^{\prime}, \alpha$, $\gamma$ and $C_{\alpha, \gamma}$.

Let $v: M \rightarrow \mathbb{R}^{d}$ be a bounded observable and $v_{n}=\sum_{k=0}^{n-1} v \circ T^{k}$. Denote $|v|_{\infty}=$ $\sup _{x \in M}|v(x)|$.

Remark 2.4. $\left|v_{n}(x)-v_{n}(y)\right| \leq 2|v|_{\infty} s(x, y)$ for all $x, y \in M$ and $n \geq 0$.

Let $\rho_{j}, j=1,2$ be two forward regular probability measures with disintegrations $\rho_{j}=\int_{E_{j}} \rho_{j, z} d \varkappa_{j}(z)$ and jump functions $r_{j}$. Let $X_{n}=\left(v_{n}, \rho_{1}\right)$ and $Y_{n}=\left(v_{n}, \rho_{2}\right)$ be the related random processes.

Theorem 2.5. The processes $\left\{X_{n}, n \geq 0\right\}$ and $\left\{Y_{n}, n \geq 0\right\}$ can be redefined on the same probability space $(\Omega, \mathbb{P})$ such that $Z=\sup _{n>0}\left|X_{n}-Y_{n}\right|$ is finite with probability one. Also:

(a) (Weak polynomial moments) If $\varkappa_{1}\left(r_{1} \geq n\right) \leq C_{\beta} n^{-\beta}, \varkappa_{2}\left(r_{2} \geq n\right) \leq C_{\beta} n^{-\beta}$ and $\mu(\tau \geq n) \leq C_{\beta} n^{-\beta}$ for all $n$ with some constants $C_{\beta}>0$ and $\beta>1$, then

$$
\mathbb{P}(Z \geq x) \leq C x^{-\beta} \quad \text { for all } x>0
$$

where the constant $C$ depends continuously (only) on $\lambda, K, R^{\prime}, \beta, C_{\beta}$ and $|v|_{\infty}$.

(b) (Strong polynomial moments) If $\int r_{1}^{\beta} d \varkappa_{1} \leq C_{\beta}, \int r_{2}^{\beta} d \varkappa_{2} \leq C_{\beta}$ and $\int \tau^{\beta} d \mu \leq C_{\beta}$ with some constants $C_{\beta}>0$ and $\beta>1$, then

$$
\int Z^{\beta} d \mathbb{P} \leq C
$$

where the constant $C$ depends continuously (only) on $\lambda, K, R^{\prime}, \beta, C_{\beta}$ and $|v|_{\infty}$. 
(c) (Exponential and stretched exponential moments) If $\varkappa_{1}\left(r_{1} \geq n\right) \leq C_{\alpha, \gamma} e^{-\alpha n^{\gamma}}$, $\varkappa_{2}\left(r_{2} \geq n\right) \leq C_{\alpha, \gamma} e^{-\alpha n^{\gamma}}$ and $\mu(\tau \geq n) \leq C_{\alpha, \gamma} e^{-\alpha n^{\gamma}}$ for all $n$ with some constants $\alpha>0, \gamma \in(0,1], C_{\alpha, \gamma}>0$, then

$$
\mathbb{P}(Z \geq x) \leq C e^{-A x^{\gamma}} \quad \text { for all } x>0,
$$

where the constants $C>0$ and $A>0$ depend continuously (only) on $\lambda, K, R^{\prime}, \alpha$, $\gamma, C_{\alpha, \gamma}$ and $|v|_{\infty}$.

Proofs of Theorems 2.3 and 2.5 are in Section 4 .

\section{Applications}

\subsection{Lévy-Prokhorov and Wasserstein distances}

Let $X$ and $Y$ be $\mathbb{R}^{d}$-valued random variables, and $\mathbb{P}_{X}, \mathbb{P}_{Y}$ be the associated probability measures on $\mathbb{R}^{d}$. Recall the following definitions:

Definition 3.1. The Lévy-Prokhorov distance between $X$ and $Y$ is

$$
\begin{gathered}
d_{L P}(X, Y)=\inf \left\{\varepsilon>0: \mathbb{P}_{X}(A) \leq \mathbb{P}_{Y}\left(A^{\varepsilon}\right)+\varepsilon \text { and } \mathbb{P}_{Y}(A) \leq \mathbb{P}_{X}\left(A^{\varepsilon}\right)+\varepsilon\right. \\
\text { for all Borel } \left.A \subset \mathbb{R}^{d}\right\}
\end{gathered}
$$

where $A^{\varepsilon}=\left\{x: \inf _{y \in A}|x-y| \leq \varepsilon\right\}$.

Definition 3.2. For $p \geq 1$, the $p^{\text {th }}$ Wasserstein distance between $X$ and $Y$ is

$$
d_{W, p}(X, Y)=\inf \left[\mathbb{E}\left(|X-Y|^{p}\right)\right]^{1 / p}
$$

where the infimum is taken over all couplings of $X$ and $Y$.

Suppose that $X_{n}$ and $Y_{n}$ are as in Theorem 2.5 (a), under the assumption of the polynomial tails. Then Theorem 2.5 implies the following:

Corollary 3.3. For each $n \geq 0$ and $1 \leq p<\beta$,

$$
d_{L P}\left(X_{n}, Y_{n}\right) \leq C_{L P} \quad \text { and } \quad d_{W, p}\left(X_{n}, Y_{n}\right) \leq C_{W, p}
$$

where the constants $C_{L P}$ and $C_{W, p}$ depend continuously (only) on $p$ and the constant $C$ from Theorem 2.5 (a). In particular, they do not depend on $n$.

Proof. Let $n$ be fixed. Theorem 2.5 provides us with a coupling of $X_{n}$ and $Y_{n}$ on a probability space $(\Omega, \mathbb{P})$ such that $Z=\left|X_{n}-Y_{n}\right|$ satisfies $\mathbb{P}(Z \geq x) \leq C x^{-\beta}$ for all $x>0$.

By definition, $d_{W, p}\left(X_{n}, Y_{n}\right) \leq\left(\mathbb{E}\left(Z^{p}\right)\right)^{1 / p}$, and the bound on $d_{W, p}\left(X_{n}, Y_{n}\right)$ follows. By [7. Theorem 2], $d_{L P}\left(X_{n}, Y_{n}\right) \leq \sqrt{d_{W, 1}\left(X_{n}, Y_{n}\right)}$.

Remark 3.4. Our estimates on the distances between $X_{n}$ and $Y_{n}$ do not depend on $n$. It follows that the distances between their normalized versions, such as $n^{-1 / 2} X_{n}$ and $n^{-1 / 2} Y_{n}$, converge to zero as $n$ goes to infinity. 


\subsection{Disintegration for the $T$-invariant measure}

Recall that $\mu$ is the absolutely continuous $F$-invariant measure. Following [23], there exists a unique $T$-invariant ergodic probability measure $\rho$ on $M$, with respect to which $\mu$ is absolutely continuous.

To define the regular measures, we fix $R^{\prime}>K \lambda /(\lambda-1)$. Here we show that $\rho$ fits the setup of Theorems 2.3 and 2.5 :

Proposition 3.5. The measure $\rho$ is forward regular: $\rho=\int_{E} \rho_{z} d \varkappa(z)$, with jump function $r: E \rightarrow \mathbb{N}_{0}$ such that $\varkappa(r=n)=\bar{\tau}^{-1} \mu(\tau \geq n)$, where $\bar{\tau}=\int_{Y} \tau d \mu$.

Proof. We start by constructing a Young tower $\breve{M}=\{(y, \ell) \in Y \times \mathbb{Z}: 0 \leq \ell<\tau(y)\}$ with the tower map

$$
\breve{T}(y, \ell)=\left\{\begin{array}{ll}
(y, \ell+1), & \ell<\tau(y)-1, \\
(F y, 0), & \ell=\tau(y)-1
\end{array} .\right.
$$

The projection $\pi: \breve{M} \rightarrow M, \pi(y, \ell)=T^{\ell}(y)$ serves as a semiconjugacy between $\breve{T}$ and $T$. The natural probability measure $\breve{\rho}=\mu \times$ counting on $\breve{M}$ is $\breve{T}$-invariant, and its projection $\rho=\pi_{*} \breve{\rho}$ is the only $T$-invariant ergodic probability measure $M$ such that $\mu \ll \rho$.

Using the definition of $\breve{\rho}$ and $\pi$, we can write $\rho$ as

$$
\rho=\bar{\tau}^{-1} \sum_{a \in \alpha} \sum_{\ell=0}^{\tau(a)-1} \mu(a) T_{*}^{\ell} \mu_{a},
$$

where $\mu_{a}$ is the normalized restriction of $\mu$ to $a$, i.e. $\mu_{a}(S)=(\mu(a))^{-1} \mu(a \cap S)$ for all $S \subset M$.

Let $E=\{(a, \ell) \in \alpha \times \mathbb{Z}: 0 \leq \ell<\tau(a)\}$ and $\varkappa(a, \ell)=\bar{\tau}^{-1} \mu(a)$. Then $\varkappa$ is a probability measure on $E$, and

$$
\rho=\sum_{(a, \ell) \in E} \rho_{a, \ell} \varkappa(a, \ell), \quad \text { where } \rho_{a, \ell}=T_{*}^{\ell} \mu_{a}
$$

is the disintegration we are after. Further, let $r: E \rightarrow \mathbb{Z}, r(a, \ell)=\tau(a)-\ell$. Then for every $a, \ell$, the measure $T_{*}^{r(a, \ell)} \rho_{a, \ell}=F_{*} \mu_{a}$ is supported on $Y$, and its density is $p_{a}(y)=$ $(\mu(a))^{-1} \zeta\left(y_{a}\right)$, where $y_{a}$ is the unique preimage of $y$ in $a$ under $F$. By (11), $T_{*}^{r(a, \ell)} \rho_{a, \ell}$ is regular.

Finally,

$$
\varkappa(r=n)=\sum_{(a, \ell) \in E} 1_{\ell=\tau(a)-n} \varkappa(a, \ell)=\bar{\tau}^{-1} \sum_{a \in \alpha: \tau(a) \geq n} \mu(a)=\bar{\tau}^{-1} \mu(\tau \geq n) .
$$

\subsection{Intermittent maps}

Consider a family of Pomeau-Manneville maps, as in [15], $T:[0,1] \rightarrow[0,1]$,

$$
T(x)=\left\{\begin{array}{ll}
x\left(1+2^{\gamma} x^{\gamma}\right), & x \leq 1 / 2 \\
2 x-1, & x>1 / 2
\end{array},\right.
$$


where $\gamma \in(0,1)$ is a parameter. This is a popular example of maps with polynomial decay of correlations (sharp rate for Hölder observables is $n^{1-1 / \gamma}$ [9, 10, 19, 23]).

Let $M=[0,1]$. It is standard (see [23]) that $T$ fits the setup of Section 2 with $Y=[1 / 2,1]$, and $\tau$ being the first return time to $Y$.

We consider three natural probability measures on $M$ :

- $m$, the Lebesgue measure,

- $\rho$, the unique absolutely continuous measure,

- $\mu$, the absolutely continuous invariant measure for the induced map, as in Section 2 .

Let $v: M \rightarrow \mathbb{R}^{d}$ be a bounded observable, $v_{n}=\sum_{k=0}^{n-1} v \circ T^{k}$, and $X_{m, n}=\left(v_{n}, m\right)$, $X_{\rho, n}=\left(v_{n}, \rho\right)$ and $X_{\mu, n}=\left(v_{n}, \mu\right)$ be the corresponding random processes.

Theorem 3.6. The processes $\left\{X_{m, n}, n \geq 0\right\},\left\{X_{\mu, n}, n \geq 0\right\}$ and $\left\{X_{\rho, n}, n \geq 0\right\}$ can be redefined on the same probability space $(\Omega, \mathbb{P})$ so that

- $Z_{m, \mu}=\sup _{n \geq 0}\left|X_{m, n}-X_{\mu, n}\right|$ satisfies $\mathbb{P}\left(Z_{m, \mu} \geq x\right) \leq C x^{-1 / \gamma}$ for $x>0$.

- $Z_{m, \rho}=\sup _{n \geq 0}\left|X_{m, n}-X_{\rho, n}\right|$ satisfies $\mathbb{P}\left(Z_{m, \rho} \geq x\right) \leq C x^{-1 / \gamma+1}$ for $x>0$.

- $Z_{\rho, \mu}=\sup _{n>0}\left|X_{\rho, n}-X_{\mu, n}\right|$ satisfies $\mathbb{P}\left(Z_{\rho, \mu} \geq x\right) \leq C x^{-1 / \gamma+1}$ for $x>0$.

The constant $C$ depends continuously (only) on $\gamma$ and $|v|_{\infty}$.

Proof. We write $a \ll b$, if there is a constant $C$ which depends continuously only on $\gamma$ such that $a \leq C b$.

It is enough to show that with an appropriate choice of the constant $R^{\prime}$ in Definition 2.1, the measures $m$ and $\rho$ are forward regular:

(a) $m=\int_{E_{m}} m_{z} d \varkappa_{m}(z)$ with $r_{m}: E_{m} \rightarrow \mathbb{N}_{0}$ for which $T_{*}^{r_{m}(z)} m_{z}$ are regular probability measures. Also, $\varkappa_{m}\left(r_{m} \geq n\right) \ll n^{-1 / \gamma}$ for all $n>0$.

(b) $\rho=\int_{E_{\rho}} \rho_{z} d \varkappa_{\rho}(z)$ with $r_{\rho}: E_{\rho} \rightarrow \mathbb{N}_{0}$ for which $T_{*}^{r_{\rho}(z)} \rho_{z}$ are regular probability measures. Also, $\varkappa_{\rho}\left(r_{\rho} \geq n\right) \ll n^{-1 / \gamma+1}$ for all $n>0$.

Then the results follow from Theorem 2.5 and Lemma A.1.

We use the bound $\mu(\tau \geq n) \ll n^{-1 / \gamma}$, (see [11] for the proof with uniform constants). By Proposition 3.5, $\rho$ is forward regular and

$$
\varkappa_{\rho}\left(r_{\rho} \geq n\right)=\sum_{k \geq n} \varkappa_{\rho}\left(r_{\rho}=k\right)=\bar{\tau}^{-1} \sum_{k \geq n} \mu(\tau \geq k) \ll n^{-1 / \gamma+1} .
$$

This proves (b). Further we prove (a).

We extend $\tau: Y \rightarrow \mathbb{N}$ to $\tau: M \rightarrow \mathbb{N}$ by $\tau(x)=\min \left\{k \geq 1: T^{k}(x) \in Y\right\}$, and accordingly set $F: M \rightarrow Y, F(x)=T^{\tau(x)}(x)$, extending the previous definition.

It is standard [1] that $M$ can be partitioned (modulo a zero measure set) into countably many subintervals $\left[a_{k}, b_{k}\right], k \in \mathbb{N}$, on which $\tau$ is constant, and $F:\left[a_{k}, b_{k}\right] \rightarrow Y$ is a diffeomorphism with bounded distortions, i.e.

$$
\left|\log \frac{F^{\prime}(x)}{F^{\prime}(y)}\right| \ll|F(x)-F(y)| \quad \text { for all } k \text { and } x, y \in\left[a_{k}, b_{k}\right] .
$$

Further, $m(\tau \geq n) \ll n^{-1 / \gamma}$.

Let $m_{k}$ denote the normalized Lebesgue measure on $\left[a_{k}, b_{k}\right]$. It follows from (41) and (1) that $F_{*} m_{k}$ is a regular measure with $R^{\prime}$ depending continuously (only) on $\gamma$.

It follows that $m$ is forward regular: $m=\sum_{k \in \mathbb{N}} \varkappa_{m}(k) m_{k}$, with the probability space $\left(\mathbb{N}, \varkappa_{m}\right), \varkappa_{m}(k)=\left|b_{k}-a_{k}\right|$, and $r_{m}: \mathbb{N} \rightarrow \mathbb{N}_{0}, r_{m}(k)=\left.\tau\right|_{\left[a_{k}, b_{k}\right]}$.

Finally, observe that $\varkappa_{m}\left(r_{m} \geq n\right) \ll n^{-1 / \gamma}$. 


\subsection{Almost sure invariance principle}

Let $v: M \rightarrow \mathbb{R}^{d}$, and $v_{n}=\sum_{k=0}^{n-1} v \circ T^{k}$. Recall that $\mu$ is the absolutely continuous $F$-invariant measure on $Y$. Let $\rho$ be the $T$-invariant measure on $M$ as in Subsection 3.2. Suppose that $\int_{M} v d \rho=0$. Let $X_{n}=\left(v_{n}, \rho\right)$ and $Y_{n}=\left(v_{n}, \mu\right)$.

Under the assumptions that $\tau \in L^{p}, p>2$ and $v$ is Hölder continuous, Melbourne and Nicol prove in [16, 17] the ASIP for $Y_{n}$ (with rates), and claim the ASIP for $X_{n}$. However, their argument does not cover the transition from $Y_{n}$ to $X_{n}$. Here we close this gap.

Theorem 3.7. The ASIP for $X_{n}$ is equivalent to the ASIP for $Y_{n}$, with the same rates.

Remark 3.8. In [16, 17, the authors prove the ASIP for nonuniformly expanding systems and then extend the result to nonuniformly hyperbolic systems [16, Section 3]. In Theorem 3.7, $T$ is a nonuniformly expanding system, but proving it, we close the gap in both situations.

Proof of Theorem 3.7. Assume the ASIP for $X_{n}$ as in Definition 1.1, with a Brownian motion $W_{n}$ and rate $o\left(n^{\beta}\right)$.

Proposition 3.5 allows us to use Theorem 2.5 to redefine the processes $\left\{X_{n}, n \geq 0\right\}$ and $\left\{Y_{n}, n \geq 0\right\}$ on the same probability space so that $\sup _{n \geq 0}\left|X_{n}-Y_{n}\right|$ is finite almost surely.

Using Lemma A.1, we can redefine $\left\{X_{n}, n \geq 0\right\},\left\{Y_{n}, n \geq 0\right\}$ and $W_{t}$ on the same probability space so that $\sup _{n>0}\left|X_{n}-Y_{n}\right|<\infty$ and $X_{n}=W_{n}+o\left(n^{\beta}\right)$ almost surely. Then also $Y_{n}=W_{n}+o\left(n^{\beta}\right)$ almost surely.

We proved that the ASIP for $X_{n}$ implies the ASIP for $Y_{n}$, with the same rates. The same argument proves the other direction.

\section{Proof of Theorems 2.3 and 2.5}

\subsection{Outline of the proof}

Recall that $\mu$ is the absolutely continuous probability measure, invariant under the induced map $F$. To prove Theorem 2.3 , we:

(a) Build (Subsection 4.2) a countable probability space $\mathcal{A}$ with a function $t: \mathcal{A} \rightarrow \mathbb{N}_{0}$ and show that if $\rho$ is a probability measure such that $T_{*}^{n} \rho$ is regular for some $n \geq 0$, then $\rho$ has a representation

$$
\rho=\sum_{a \in \mathcal{A}} \mathbb{P}(a) \rho_{k} \quad \text { with } T_{*}^{n+t(a)} \rho_{a}=\mu \text { for all } a,
$$

where $\mathbb{P}$ is a probability measure on $\mathcal{A}$. (C.f. regenerative partition of unity in [24]).

(b) Show that the tails $\mathbb{P}(t \geq n)$ can be bounded uniformly for all regular measures (Subsection 4.3).

(c) Consider a particularly simple case, when $\rho$ is such that $T_{*}^{n} \rho=\mu$ for some $n \geq 0$. Then we take $\hat{\rho}=\left(U_{n}\right)_{*} \rho$, where $U_{n}: M \rightarrow M \times M, U_{n}(x)=\left(x, T^{n} x\right)$. We observe that the marginals of $\hat{\rho}$ on the first and second coordinates are $\rho$ and $\mu$ respectively and $\hat{\rho}(s \geq n)=0$. 
(d) The procedure in (c) transparently extends to weighted sums of measures, as in (5). We take

$$
\hat{\rho}=\sum_{a \in \mathcal{A}} \mathbb{P}(a)\left(U_{n+t(a)}\right)_{*} \rho_{a} .
$$

Observe that then $\hat{\rho}(s \geq n+k) \leq \mathbb{P}(t \geq k)$ for all $k \geq 0$.

(e) Now, (a) and (d) already prove Theorem 2.3 for the case when $T_{*}^{n} \rho$ is regular. In Subsection 4.5] we extend this to the class of all forward regular measures.

The idea of the proof of Theorem 2.5 is that if $\rho_{1}$ and $\rho_{2}$ are forward regular measures, then each of them can be coupled with $\mu$ in the sense of Theorem 2.3 . Then we couple $\rho_{1}$ and $\rho_{2}$ through their couplings with $\mu$ by a standard argument in Probability Theory, see Appendix A.

\subsection{Disintegration}

Let $P: L^{1}(Y) \rightarrow L^{1}(Y)$ be the transfer operator corresponding to $F$ and $\mu$, so $\int_{Y} P \phi \psi d \mu=\int_{Y} \phi \psi \circ F d \mu$ for all $\phi \in L^{1}$ and $\psi \in L^{\infty}$. Then $P \phi$ is given explicitly by

$$
(P \phi)(y)=\sum_{a \in \alpha} \zeta\left(y_{a}\right) \phi\left(y_{a}\right)
$$

where $y_{a}$ is the unique preimage of $y$ under $F$ lying in $a$.

Recall that $R^{\prime}$ is a fixed constant, and $R^{\prime}>K \lambda /(\lambda-1)$. Let $R=\lambda\left(R^{\prime}-K\right)$. Then $R>K+\lambda^{-1} R$. Choose $\xi \in\left(0, e^{-R}\right)$ such that $R\left(1-\xi e^{R}\right) \geq K+\lambda^{-1} R$.

Proposition 4.1. Assume that $\phi: Y \rightarrow(0, \infty)$ is such that $|\phi|_{d, \ell} \leq R^{\prime}$. Then $\phi=$ $\xi \int_{Y} \phi d \mu+\psi$, where $|\psi|_{d, \ell} \leq R$. In addition, $\left|P\left(1_{a} \psi\right)\right|_{d, \ell} \leq R^{\prime}$ for every $a \in \alpha$.

Proof. See [13, Propositions 3.1 and 3.2].

Let $\mathcal{A}$ denote the countable set of all finite words in the alphabet $\alpha$, including the empty word. For $a \in \mathcal{A}$, let $[a]$ denote the subset of words in $\mathcal{A}$ which begin with $a$. Let $\ell(a)$ denote the length of $a$. Define $t: \mathcal{A} \rightarrow \mathbb{Z}, t(a)=\sum_{k=1}^{\ell(a)} \tau\left(a_{k}\right)$, where $a_{k}$ is the $k$-th letter of $a$.

Proposition 4.2. Let $\rho$ be a probability measure on $M$ such that $T_{*}^{n} \rho$ is regular for some $n \geq 0$. Then there is a decomposition $\rho=\xi \rho^{\prime}+\sum_{a \in \alpha} r_{a} \rho_{a}$, where $\rho^{\prime}$ and all $\rho_{a}$ are probability measures and $r_{a}>0$, such that

- $e^{-R}(1-\xi) \mu(a) \leq r_{a} \leq e^{R}(1-\xi) \mu(a)$,

- $T_{*}^{n} \rho^{\prime}=\mu$,

- $T_{*}^{n+\tau(a)} \rho_{a}$ is a regular measure for every $a \in \alpha$.

Proof. Let $\chi=T_{*}^{n} \rho$. Since $\chi$ is regular probability measure, there exists $\phi: Y \rightarrow(0, \infty)$ such that $|\phi|_{d, \ell} \leq R^{\prime}, d \chi=\phi d \mu$ and $\int_{Y} \phi d \mu=1$.

By Proposition 4.1, $\phi=\xi+\psi$, where $|\psi|_{d, \ell} \leq R$. For $a \in \alpha$, define $r_{a}=\int_{a} \psi d \mu$ and $\psi_{a}=r_{a}^{-1} 1_{a} \psi$. Then $\int_{Y} \psi_{a} d \mu=1$ and by Proposition 4.1, $\left|P \psi_{a}\right|_{d, \ell} \leq R^{\prime}$. Define $\chi_{a}$ to be a probability measure on $M$ given by $d \chi_{a}=\psi_{a} d \mu$. Then $T_{*}^{\tau(a)} \chi_{a}$ is a regular probability measure with density $P \psi_{a}$. 
Observe that

$$
\chi=\xi \mu+\sum_{a \in \alpha} r_{a} \chi_{a}
$$

By [13, (3.1)],

$$
e^{-R}(1-\xi)=e^{-R} \int_{Y} \psi d \mu \leq \psi \leq e^{R} \int_{Y} \psi d \mu=e^{R}(1-\xi) .
$$

Therefore $e^{-R}(1-\xi) \mu(a) \leq r_{a} \leq e^{R}(1-\xi) \mu(a)$.

Now we use (6) to decompose $\rho$ similarly. Define $\rho^{\prime}$ to be a measure on $M$ given by $\frac{d \rho^{\prime}}{d \rho}=\frac{d \mu}{d \chi} \circ T^{n}$. Then $T_{*}^{n} \rho^{\prime}=\mu$. Similarly define $\rho_{a}, a \in \alpha$ by $\frac{d \rho_{a}}{d \rho}=\frac{d \chi_{a}}{d \chi} \circ T^{n}$. Then $T_{*}^{n} \rho_{a}=\chi_{a}$. Finally note that $\rho=\xi \rho^{\prime}+\sum_{a \in \alpha} r_{a} \rho_{a}$.

Lemma 4.3. Let $n \geq 0$ and $\rho$ be a probability measure on $M$ such that $T_{*}^{n} \rho$ is regular. There exists a probability measure $\mathbb{P}$ on $\mathcal{A}$ and a disintegration

$$
\rho=\sum_{a \in \mathcal{A}} \mathbb{P}(a) \rho_{a}
$$

where $\rho_{a}, a \in \alpha$ are probability measures on $M$ such that $T_{*}^{n+t(a)} \rho_{a}=\mu$. The measure $\mathbb{P}$ satisfies

$$
\begin{gathered}
\mathbb{P}(\ell=k)=(1-\xi)^{k} \xi \\
e^{-R}(1-\xi) \mu\left(a_{k+1}\right) \leq \mathbb{P}\left(\left[a_{1} \cdots a_{k+1}\right] \mid\left[a_{1} \cdots a_{k}\right]\right) \leq e^{R}(1-\xi) \mu\left(a_{k+1}\right),
\end{gathered}
$$

for all $k \geq 0$ and $a_{1}, \ldots, a_{k+1} \in \alpha$.

Proof. Write $\rho=\xi \rho^{\prime}+\sum_{x \in \alpha} r_{x} \rho_{x}$ as in Proposition 4.2, Then for each $x \in \alpha$ apply Proposition 4.2 again and write $\rho_{x}=\xi \rho_{x}^{\prime}+\sum_{y \in \alpha} r_{x y} \rho_{x y}$. Apply the same to each $\rho_{x y}$ and so on. Then

$$
\rho=\xi \rho^{\prime}+\sum_{x \in \alpha} r_{x} \xi \rho_{x}^{\prime}+\sum_{x, y \in \alpha} r_{x} r_{x y} \xi \rho_{x y}^{\prime}+\cdots
$$

This is a disintegration as in (7) with $\mathbb{P}(a)=r_{a_{1}} r_{a_{1} a_{2}} \cdots r_{a_{1} a_{2} \cdots a_{n}} \xi$ for $a=a_{1} \cdots a_{n} \in \mathcal{A}$. Conditions (8) are immediate.

\subsection{Polynomial and exponential tails}

Let $\rho$ be a measure as in Lemma 4.3 and $\mathbb{P}$ be the corresponding measure on $\mathcal{A}$. Recall that $t: \mathcal{A} \rightarrow \mathbb{Z}$ is the word length.

In this subsection we obtain elementary estimates of moments of $t$ in situations when $\int_{Y} \tau^{p} d \mu<\infty$ for some $p>1$, or $\int_{Y} e^{\gamma \tau} d \mu<\infty$ for some $\gamma>0$.

For $n \geq 1$, let $\mathcal{A}_{n}$ be the subset of $\mathcal{A}$ of all words of length $n$. By Lemma 4.3, $\mathbb{P}\left(\mathcal{A}_{n}\right)=(1-\xi)^{n} \xi$. Let $\mathbb{P}_{n}$ denote the conditional probability measure on $\mathcal{A}_{n}$.

Elements of $\mathcal{A}_{n}$ have the form $a=a_{1} \cdots a_{n}$, and $a_{1}, \ldots, a_{n}$ can be considered as random variables with values in $\alpha$, and $t=\tau\left(a_{1}\right)+\cdots+\tau\left(a_{n}\right)$.

It follows from Lemma 4.3 that for all $k \leq n$ and $x \in \alpha$,

$$
\mathbb{P}_{n}\left(a_{k}=x \mid a_{1}, \ldots, a_{k-1}\right) \leq e^{R} \mu(x)
$$




\subsubsection{Polynomial tails}

Proposition 4.4. Suppose that there exist $C_{\tau}>0$ and $\beta>1$ such that $m(\tau \geq \ell) \leq C_{\tau} \ell^{-\beta}$ for $\ell \geq 1$. Then $\mathbb{P}(t \geq \ell) \leq C \ell^{-\beta}$, where the constant $C>0$ depends continuously on $R$, $\xi$ and $C_{\tau}$.

Proof. Let $k \leq n$, and $a=a_{1} \cdots a_{n} \in \mathcal{A}_{n}$. By (9),

$$
\mathbb{P}_{n}\left(\tau\left(a_{k}\right) \geq \ell\right) \leq e^{R} m(\tau \geq \ell) \leq C_{\tau} e^{R} \ell^{-\beta} .
$$

Next,

$$
\mathbb{P}_{n}(t \geq \ell) \leq \sum_{k=1}^{n} \mathbb{P}_{n}\left(\tau\left(a_{k}\right) \geq \ell / n\right) \leq n C_{\tau} e^{R}(\ell / n)^{-\beta}
$$

Finally,

$$
\mathbb{P}(t \geq \ell)=\sum_{n=1}^{\infty} \mathbb{P}\left(\mathcal{A}_{n}\right) \mathbb{P}_{n}(t \geq \ell) \leq C_{\tau} e^{R} \xi \ell^{-\beta} \sum_{n=1}^{\infty}(1-\xi)^{n} n^{1+\beta}
$$

Proposition 4.5. Suppose that there exist $C_{\tau}>0$ and $\beta>1$ such that $\int \tau^{\beta} d m \leq C_{\tau}$. Then $\int t^{\beta} d \mathbb{P} \leq C$, where the constant $C>0$ depends continuously on $R, \xi$ and $C_{\tau}$.

Proof. Let $k \leq n$, and $a=a_{1} \cdots a_{n} \in \mathcal{A}_{n}$. By (9),

$$
\int \tau^{\beta}\left(a_{k}\right) d \mathbb{P}_{n} \leq e^{R} \int \tau^{\beta} d m \leq C_{\tau} e^{R}
$$

Next,

$$
t^{\beta}(a)=\left(\tau\left(a_{1}\right)+\cdots+\tau\left(a_{n}\right)\right)^{\beta} \leq n^{\beta-1}\left(\tau^{\beta}\left(a_{1}\right)+\cdots+\tau^{\beta}\left(a_{n}\right)\right),
$$

thus

$$
\int t^{\beta} d \mathbb{P}_{n} \leq n^{\beta-1} C_{\tau} e^{R}
$$

Finally,

$$
\int t^{\beta} d \mathbb{P}=\sum_{n=1}^{\infty} \mathbb{P}\left(\mathcal{A}_{n}\right) \int t^{\beta} d \mathbb{P}_{n} \leq C_{\tau} e^{R} \xi \sum_{n=1}^{\infty}(1-\xi)^{n} n^{\beta-1} .
$$

\subsection{2 (Stretched) exponential tails}

Proposition 4.6. Let $X_{1}, \ldots, X_{n}$ be nonnegative random variables. Suppose that there exist $\alpha>0, \gamma \in(0,1]$, such that

$$
\mathbb{P}\left(X_{k} \geq \ell \mid X_{1}=x_{1}, \ldots, X_{k-1}=x_{k-1}\right) \leq C e^{-\alpha \ell^{\gamma}}
$$

for all $\ell \geq 0,1 \leq k \leq n$ and $x_{1}, \ldots, x_{k-1} \geq 0$. Then for all $A \in(0, \alpha / 2], \ell \geq 0$,

$$
\mathbb{P}\left(X_{1}+\cdots+X_{n} \geq \ell\right) \leq\left(1+A C_{1}\right)^{n} e^{-A \ell^{\gamma}},
$$

where $C_{1}$ depends continuously on $C, \gamma$ and $\alpha$. 
Proof. See [13, Proposition 4.11].

Proposition 4.7. Suppose that there exist $C_{\tau}>0, \alpha>0$ and $\gamma \in(0,1]$ such that $m(\tau \geq \ell) \leq C_{\tau} e^{-\alpha \ell^{\gamma}}$ for $\ell \geq 1$. Then $\mathbb{P}(t \geq \ell) \leq C e^{-A \ell^{\gamma}}$, where the constants $C>0$ and $A \in(0, \alpha)$ depend continuously on $R, \xi, C_{\tau}, \alpha$ and $\gamma$.

Proof. Let $k \leq n$, and $a=a_{1} \cdots a_{n} \in \mathcal{A}_{n}$. By (9),

$$
\mathbb{P}_{n}\left(\tau\left(a_{k}\right) \geq \ell \mid a_{1}, \ldots, a_{k-1}\right) \leq e^{R} m(\tau \geq \ell) \leq C_{\tau} e^{-\alpha \ell^{\gamma}} .
$$

By Proposition 4.6,

$$
\mathbb{P}_{n}(t \geq \ell) \leq\left(1+A C_{1}\right)^{n} e^{-A \ell^{\gamma}}
$$

for all $A \in(0, \alpha / 2)$. Taking $A$ small enough, we obtain

$$
\mathbb{P}(t \geq \ell)=\sum_{n=1}^{\infty} \mathbb{P}\left(\mathcal{A}_{n}\right) \mathbb{P}_{n}(t \geq \ell) \leq \xi e^{-A \ell^{\gamma}} \sum_{n=1}^{\infty}(1-\xi)^{n}\left(1+A C_{1}\right)^{n}=C e^{-A \ell^{\gamma}}
$$

with $C<\infty$.

\subsection{Coupling}

Recall that $s: M \times M \rightarrow \mathbb{N}_{0} \cup\{\infty\}$ is defined by

$$
s(x, y)=\inf \left\{\max \{k, n\}: k, n \geq 0, T^{k} x=T^{n} y\right\} .
$$

Lemma 4.8. Let $n \geq 0$ and $\rho$ be a probability measure on $M$ such that $T_{*}^{n} \rho$ is regular. Then there exists a measure $\hat{\rho}$ on $M \times M$ with marginals $\rho$ and $\mu$ on the first and second coordinates respectively, such that $s(x, y)<\infty$ for $\hat{\rho}$-almost every $(x, y) \in M \times M$.

If there exist $C_{\tau}>0$ and $\beta>1$ such that $m(\tau \geq \ell) \leq C_{\tau} \ell^{-\beta}$ for $\ell \geq 1$, then $\hat{\rho}(s \geq \ell) \leq C(\ell-n)^{-\beta}$ for $\ell \geq n+1$ and some constant $C>0$.

If there exist constants $C_{\tau}>0, \alpha>0$ and $\gamma \in(0,1]$ such that $m(\tau \geq \ell) \leq C_{\tau} e^{-\alpha \ell^{\gamma}}$ for $\ell \geq 1$, then $\hat{\rho}(s \geq \ell) \leq C e^{-A(\ell-n)^{\gamma}}$ for $\ell \geq n+1$ and some constants $A \in(0, \alpha)$ and $C>0$.

In both cases above, the constants $C$ and $A$ depend continuously (only) on $R, \xi, C_{\tau}$, $\beta, \alpha$ and $\gamma$.

Proof. Lemma 4.3 provides us with the decomposition $\rho=\sum_{a \in \mathcal{A}} \mathbb{P}(a) \rho_{a}$ such that $T_{*}^{n+t(a)} \rho_{a}=\mu$ for every $a$.

For $k \geq 0$ define $U_{k}: M \rightarrow M \times M, U_{k}(x)=\left(x, T^{k} x\right)$. Define

$$
\hat{\rho}=\sum_{a \in \mathcal{A}} \mathbb{P}(a)\left(U_{n+t(a)}\right)_{*} \rho_{a} .
$$

It is clear that the marginals of $\left(U_{n+t(a)}\right)_{*} \rho_{a}$ on the first and second components are $\rho_{a}$ and $\mu$ respectively. Therefore the marginals of $\hat{\rho}$ are $\rho$ and $\mu$.

Observe that $s(x, y) \leq n+t(a)$ for $\left(U_{n+t(a)}\right)_{*} \rho_{a}$-almost every $(x, y) \in M \times M$. Thus $s<\infty$ for $\hat{\rho}$-almost every $(x, y) \in M \times M$.

It remains to estimate $\hat{\rho}(s \geq \ell)$. Note that $\hat{\rho}(s \geq \ell) \leq \mathbb{P}(t \geq \ell-n)$. The results follow directly from Propositions 4.4 and 4.7. 


\subsection{Proof of Theorem 2.3}

By Lemma 4.8, for every $z \in E$ there exists a probability measure $\hat{\rho}_{z}$ on $M \times M$ with marginals $\rho_{z}$ and $\mu$ respectively such that $s<\infty$ almost surely.

Remark 4.9. In Proposition 4.2 and Lemma 4.3, we construct the measures $\rho_{a}, a \in \mathcal{A}$ (as in Lemma 4.3) by explicit formulas, and it is a straightforward verification that, as long as $\rho_{z}$ is a measurable family, so are the respective $\rho_{z, a}$ for each $a \in \mathcal{A}$. Further, $\hat{\rho}_{z}$ are explicitly constructed from $\rho_{z, a}$ in Lemma 4.8, so the family $\hat{\rho}_{z}$ is measurable.

Define $\hat{\rho}=\int_{E} \hat{\rho}_{z} d \varkappa(z)$. Then the marginals of $\hat{\rho}$ are $\rho$ and $\mu$ respectively, and $s<\infty$ almost surely with respect to $\hat{\rho}$.

It remains to estimate the tails $\hat{\rho}(s \geq n)$. We prove the weak polynomial case, the others are similar. Using Lemma 4.8, write

$$
\begin{aligned}
\hat{\rho}(s \geq n) & =\int_{E} \hat{\rho}_{z}(s \geq n) d \varkappa(z) \ll \int_{E} \min \left\{1,(n-r(z))^{-\beta}\right\} d \varkappa(z) \\
& \leq \varkappa(r \geq n / 2)+\int_{E}(n / 2)^{-\beta} d \varkappa(z) \ll n^{-\beta} .
\end{aligned}
$$

\subsection{Proof of Theorem 2.5}

Assume without loss that $|v|_{\infty} \leq 1 / 2$.

Let $U_{n}=\left(v_{n}, \mu\right)$. It follows from Theorem 2.3 that the processes $\left\{X_{n}, n \geq 0\right\}$ and $\left\{U_{n}, n \geq 0\right\}$ can be redefined on the probability space $\left(M \times M, \hat{\rho}_{X U}\right)$ where $s<\infty$ $\hat{\rho}_{X U}$-almost surely. By Remark 2.4, $Z_{X U}=\sup _{n}\left|X_{n}-U_{n}\right| \leq s$, thus $Z_{X U}$ is also finite $\hat{\rho}_{X U}$-almost surely.

Similarly, $\left\{Y_{n}, n \geq 0\right\}$ and $\left\{U_{n}, n \geq 0\right\}$ can be redefined on $\left(M \times M, \hat{\rho}_{Y U}\right)$ with $\hat{\rho}_{Y U}$-almost surely finite $Z_{Y U}=\sup _{n}\left|Y_{n}-U_{n}\right|$.

By Lemma A.1, all three processes $\left\{X_{n}, n \geq 0\right\},\left\{Y_{n}, n \geq 0\right\}$ and $\left\{U_{n}, n \geq 0\right\}$ can be redefined on the same probability space $(\Omega, \mathbb{P})$ so that the joint distributions of pairs $\left\{\left(X_{n}, U_{n}\right), n \geq 0\right\}$ and $\left\{\left(Y_{n}, U_{n}\right), n \geq 0\right\}$ are as above. Further we work on this probability space.

Observe that $Z=\sup _{n}\left|X_{n}-Y_{n}\right| \leq Z_{X U}+Z_{Y U}$. It follows that $Z$ is almost surely finite.

It remains to estimate $\mathbb{P}(Z \geq x)$ for $x \geq 0$. The bounds follow transparently from Theorem 2.3 and the relation

$$
\begin{aligned}
\mathbb{P}(Z \geq x) & \leq \mathbb{P}\left(Z_{X U} \geq x / 2\right)+\mathbb{P}\left(Z_{Y U} \geq x / 2\right) \\
& \leq \hat{\rho}_{X U}(s \geq x / 2)+\hat{\rho}_{Y U}(s \geq x / 2) .
\end{aligned}
$$

\section{A Joining of couplings}

Suppose that $X_{j}, j=1,2,3$ are random variables on probability spaces $\Omega_{j}$ with values in some measurable spaces $R_{j}$.

Assume that $X_{1}$ and $X_{2}$ can be redefined on a new probability space $\Omega_{12}$, so that the joint distribution of $\left(X_{1}, X_{2}\right)$ has some useful property, for example that $\left|X_{1}-X_{2}\right|<1$ almost surely. 
Assume similarly that $X_{2}$ and $X_{3}$ can be redefined on a probability space $\Omega_{23}$ with a joint distribution of $\left(X_{2}, X_{3}\right)$ of interest.

Recall that a Polish space is a separable completely metrizable topological space. In this paper we work with continuous and discrete time random processes, which can be viewed as random variables with values in the space of càdlàg functions on $[0, \infty)$, or $\mathbb{R}^{\mathbb{N}}$. These spaces are Polish.

Polish spaces are universally measurable (see [20] for the definition and discussion). This is a technical but useful property, which allows to join couplings:

Lemma A.1. If all value spaces $R_{j}$ are universally measurable, then $X_{1}, X_{2}$ and $X_{3}$ can be redefined on the same probability space $\Omega_{123}$, such that the distributions of $\left(X_{1}, X_{2}\right)$ and $\left(X_{2}, X_{3}\right)$ are the same as on $\Omega_{12}$ and $\Omega_{23}$ respectively.

Proof. Note that the probability spaces, on which the random variables $X_{j}$ are defined, are irrelevant, so we can instead work directly with the corresponding probability measures on $R_{j}$. In this setting the result is proved in [20, Lemma 7].

Remark A.2. It was pointed out by the referee that there is an earlier reference [1, Lemma A.1] for the result of Lemma A.1 in case when $R_{j}$ are separable Banach spaces. It is perfectly sufficient for our purposes (c.f. [8, Subsection 3.1]) and avoids the concept of universal measurability.

We are happy to mention [1, yet we keep our Lemma A.1, because it is more general, and may be easier to use. For instance, it is not clear how to apply [1, Lemma A.1] for the space of cádlág functions with Skorokhod metric: it is separable and complete (thus Polish), but without a corresponding norm.

\section{Acknowledgements}

This research was supported in part by a European Advanced Grant StochExtHomog (ERC AdG 320977). The author is grateful to Ian Melbourne for support and numerous suggestions. The author is grateful to the anonymous referee for a very thorough review, many helpful comments and a request to adapt the manuscript for a larger audience.

\section{References}

[1] I. Berkes, W.Philipp, Approximation theorems for independent and weakly dependent random vectors, Ann. Probab. 7 (1979), 29-54.

[2] R. Bowen. Equilibrium States and the Ergodic Theory of Anosov Diffeomorphisms, Lecture Notes in Math., 470 (1975), Springer, Berlin.

[3] N. Chernov, Advanced statistical properties of dispersing billiards, J. Stat. Phys., 122 (2006), 1061-1094.

[4] N. Chernov and R. Markarian, Chaotic Billiards, Math. Surveys Monogr., 127 (2006). 
[5] C. Cuny, F. Merlevède, Strong invariance principles with rate for "reverse" martingales and applications, J. Theoret. Probab., 28 (2015), 137-183.

[6] M. Denker, W.Philipp, Approximation by Brownian motion for Gibbs measures and flows under a function, Ergodic Theory Dynam. Systems 4 (1984), 541-552.

[7] A.L. Gibbs, F.E. Su, On choosing and bounding probability metrics, Int. Stat. Rev. 70 (2002), 419-435.

[8] S. Gouëzel, Almost sure invariance principle for dynamical systems by spectral methods, Ann. Probab. 38 (2010), 1639-1671.

[9] S. Gouëzel, Sharp polynomial estimates for the decay of correlations, Israel J. Math. 139 (2004), 29-65.

[10] H. Hu, Decay of correlations for piecewise smooth maps with indifferent fixed points, Ergodic Theory Dynam. Systems 24 (2004), 495-524.

[11] A. Korepanov, Linear response for intermittent maps with summable and nonsummable decay of correlations, Nonlinearity 29 (2016), 1735-1754.

[12] A. Korepanov, Z. Kosloff and I. Melbourne, Averaging and rates of averaging for uniform families of deterministic fast-slow skew product systems, Studia Math. 238 (2017), 59-89.

[13] A. Korepanov, Z. Kosloff, I. Melbourne, Explicit coupling argument for nonuniformly hyperbolic transformations, Proc. Roy. Soc. Edinburgh Sect. A. (2016), to appear.

[14] A. Korepanov, Z. Kosloff, I. Melbourne, Martingale-coboundary decomposition for families of dynamical systems, Ann. Inst. H. Poincaré Anal. Non Linéaire (2016), to appear.

[15] C. Liverani, B. Saussol, and S. Vaienti, A probabilistic approach to intermittency, Ergodic Theory Dynam. Systems 19 (1999), 671-685.

[16] I. Melbourne, M. Nicol, Almost sure invariance principle for nonuniformly hyperbolic systems, Commun. Math. Phys. 260 (2005), 131-146.

[17] I. Melbourne, M. Nicol, A vector-valued almost sure invariance principle for hyperbolic dynamical systems, Ann. Probab. 37 (2009), 478-505.

[18] W. Philipp, W. Stout, Almost sure invariance principles for partial sums of weakly dependent random variables, Amer. Math. Soc. Mem. 161 (1975).

[19] O. Sarig, Subexponential decay of correlations, Invent. Math. 150 (2002), 629-653.

[20] R. M. Shortt, Universally measurable spaces: an invariance theorem and diverse characterizations, Fund. Math. 121 (1984), 169-176.

[21] Y. G. Sinai. Gibbs measures in ergodic theory, Russ. Math. Surv. 27 (1972) 21-70. 
[22] L.-S. Young, Statistical properties of dynamical systems with some hyperbolicity, Ann. of Math. 147 (1998), 585-650.

[23] L.-S. Young, Recurrence times and rates of mixing, Israel J. Math. 110 (1999), 153188.

[24] R. Zweimüller, Measure preserving transformations similar to Markov shifts, Israel J. Math. 173 (2009), 421-443. 